\title{
Las actividades periescolares durante el primer peronismo: estrategias socio-pedagógicas para el cuidado de la infancia
}

The extracurricular activities during the first Peronism: social and pedagogical strategies for the care of the childhood

\author{
Gabriela Verónica Ferreyra \\ Universidad Nacional de Tres de Febrero, Argentina \\ gferreyra@untref.edu.ar
}

\section{Resumen:}

El objetivo de este artículo es analizar el programa de actividades periescolares que funcionó en las escuelas primarias de la actual ciudad de Buenos Aires entre 1948 y 1950. El corpus documental, compuesto por fotografías y relatos escritos por el personal docente, revela que el gobierno peronista recuperó las políticas sociales que se fomentaron en la Argentina durante los primeros años del siglo XX en las instituciones educativas y las adaptó en un fugaz proyecto que amalgamó los objetivos oficiales de protección de la infancia y la familia, la democratización de la cultura y la aplicación de las reformas pedagógicas.

Palabras clave: Primer peronismo, Infancia, Escuela primaria, Actividades periescolares.

\section{AbStract:}

The aim of this paper is to analyze programme of extracurricular activities implemented in the elementary school of the actual city of Buenos Aires between 1948 and 1950. The documentary corpus, composed of written reports and pictures recorded by the teachers, shows that the Peronist government regained the social policies that were fomented in the Argentina during the first years of the 20th century in the public school and adapted them in a project that gathered the aim of protecting children and family, the democratization of culture and la and education reform.

KeYwords: First Peronism, Childhood, Elementary school, Extracurricular activities.

\section{INTRODUCCIÓN}

En el marco de las políticas sociales dirigidas hacia la población infantil, las propuestas de difusión de las manifestaciones culturales destinadas a los sectores populares y los ensayos asistencialistas realizados desde principios del siglo XX por diferentes instituciones, las escuelas y los maestros fueron requeridos durante el primer gobierno de Perón (1946-1952) para coordinar y sistematizar la articulación entre la ayuda social y la formación integral del alumnado. La reforma educativa para la escuela primaria, decretada en 1947 y aplicada a partir de 1948, proyectó la revisión y escritura de un nuevo programa de estudios, la creación de cursos e instituciones de capacitación técnico-pedagógica para el magisterio y la apertura de los edificios escolares para cubrir espacios de recreación y contención destinados a la comunidad. En ese contexto, entre 1948 y 1950 se puso en marcha un programa de conferencias y charlas para las familias, cursos de pre-aprendizaje y Clubes Escolares que recibieron el nombre de actividades periescolares. ${ }^{1}$ Consideramos que a través de estas prácticas concretas, el gobierno peronista recuperó los proyectos socio-educativos previos que no habían tenido éxito sostenido en las escuelas y los resignificó al inscribirlos en el marco de los objetivos oficiales de protección de la infancia y la familia, la democratización de la cultura y la reforma educativa establecida en el Primer Plan Quinquenal (1947-1952). 
El propósito de este artículo es reconstruir el funcionamiento de las experiencias que se llevaron a cabo en las escuelas primarias de la actual ciudad de Buenos Aires durante los primeros años peronistas y analizar los argumentos que ofrecieron los funcionarios para aplicarlas. Para estudiar las funciones y características se ha recurrido al archivo de memorias escolares que se encuentra en la biblioteca Joaquín V. González del Instituto Bernasconi de la Ciudad de Buenos Aires. Los documentos, solicitados por el supervisor de cada Consejo Escolar (C.E.) de la capital federal, contienen informes, fotografías, dibujos y folletos, que describen las características del barrio, el período de fundación de cada institución, los nombres del personal y una recopilación de las actividades que allí se desarrollaron. ${ }^{2}$

El trabajo está organizado en dos apartados, el primero trata sobre los argumentos sociales y pedagógicos de los funcionarios del área educativa que dieron marco a la reformulación del rol de la escuela primaria en la protección del niño-alumno. En la segunda parte, se analizan los espacios escolares dedicados a las familias, Asociaciones para Padres y cursos de Pedagogía Familiar, y los destinados a los niños, cursos de preaprendizaje y Clubes Escolares.

\section{El CUIDADo DE LA POBLACión INFANTIL DURANTE EL PERONISMO: LA ESCUELA Y LA PROTECCIÓN DEL NIÑO-ALUMNO}

En la Argentina, la historiografía sobre la infancia ha abordado la cuestión desde una variedad de enfoques, problemas y fuentes que analizan la niñez en términos de "expresión cultural particular, histórica, políticamente contingente y sujeta a cambios" (Cosse, Llobet, Villalta y Zapiola, 2011, p. 12). ${ }^{3}$ En el campo de los estudios sobre los años peronistas, se han analizado las instituciones, discursos, actores sociales y experiencias impulsadas por el gobierno en clave de continuidad y cambio con la intervención estatal surgida en la década del treinta. Los autores han analizado la niñez en relación a las políticas sociales de la primera parte del siglo XX (Lionetti, 2009; Lvovich y Suriano, 2006; Aversa 2008), el papel de la familia (Moreno y Cacopardo, 2005-2006; Cosse, 2018) y las políticas e instituciones sanitarias creadas durante el período (Biernat y Ramacciotti, 2013; Ramacciotti, 2010; Billorou, 2017), los discursos y la política partidaria (Carli, 2002 y 2005; Girbal-Blacha, 2006), la cultura, el entretenimiento y las reformas en educación (Plotkin, 2007; Bordagaray y Gorza, 2010; Girbal-Blacha, 2014 y Leonardi, 2010; Cammarota, 2010; Petitti, 2017). En línea con estas últimas investigaciones, el artículo profundiza la lectura sobre el protagonismo que el Estado otorgó a la escuela en la protección del alumno-niño desde una narrativa que excedió los intereses políticos-partidarios. ${ }^{4}$

El cuidado de la población infantil ocupó un espacio central durante el primer gobierno de Perón (1946-1952). El impacto de los desastres de la Segunda Guerra, las proclamas internacionales y la exigencia de la inscripción de la niñez en una sociedad de masas, se conjugaron en el ámbito nacional, con las demandas sociales de alimentación, salud y ampliación del acceso a la escolaridad (Carli, 2002, pp. 262-266). En la Argentina, en un contexto de creciente ampliación de las capacidades estatales, los derechos de los niños cobraron un lugar central

\footnotetext{
"y se plasmaron en un conjunto de acciones que tuvo como centro la distribución de bienes y servicios por parte del Estado. La modificación del rol estatal fue vista como la ruptura de la beneficencia privada y el paso hacia la asistencia social. No se trató de un mero cambio de términos, sino de la aceptación, por parte del Estado, de su obligación de intentar resolver las variadas cuestiones que se presentaban en las diferentes regiones del territorio nacional" (Ramacciotti, 2010, p.175)
}

De ese modo, el ejecutivo puso en marcha un conjunto de medidas aplicadas bajo el nombre de justicia social. El término, que hacía referencia a la distribución equitativa de los bienes materiales, espirituales y morales, fue el argumento que justificó el aumento presupuestario y la reorientación de la función del sistema de enseñanza primaria. En ese sentido, el Estado mantuvo la intervención sobre los problemas y sus respuestas desde la categoría establecida por la Ley 1.420, el "niño en edad escolar". 
En la conferencia "Aplicación del Plan de Gobierno en la enseñanza primaria”, el Subsecretario de Instrucción Pública Jorge Pedro Arizaga definió la orientación de la reforma peronista decretada en 1947. La escuela debía ser un elemento utilizado por el Estado para la protección del niño en todas sus actividades y necesidades a través de la compensación económica a los padres, la coordinación entre los organismos escolares destinados a la ayuda social y la vigilancia del alumno sano y enfermo. Cuatro de los siete aspectos que conformaban la "Transformación del hacer docente" se referían a la vitalización de la escuela primaria, idea estrechamente relacionada con la actividad del alumno, la interacción con la comunidad y la organización de la acción periescolar (Arizaga, 1947, pp. 43 y 51). Tanto los de corte asistencial, como los de corte pedagógico, todos habían comenzado a perfilarse durante los gobiernos anteriores.

Desde fines del siglo XIX y comienzos del siglo XX, el Estado y la sociedad civil habían alentado una red asistencialista que contaba con la colaboración de asociaciones protectoras, médicos, educadores, profesores de educación física, asistentes sociales, mujeres de la elite activas en la caridad y la filantropía social, católicos sociales, socialistas, liberales, libertarios, masones y librepensadores (Armus, 2009, p. 99). En el plano escolar, en la ciudad de Buenos Aires al comenzar la década del treinta, algunas escuelas primarias contaban con asociaciones cooperadoras y grupos de ex alumnos que realizaban obras benéficas y colaboraban con los gastos del edificio y con material para el trabajo diario.

En 1938, se creó la Comisión Nacional de Ayuda Escolar (CNAE) que implementó un sistema de ayuda social a las familias carentes de recursos económicos y potenció las acciones que había realizado en el pasado el Consejo Nacional de Educación (CNE) con la creación del cuerpo médico escolar, la copa de leche y la entrega de vestidos a niños indigentes. ${ }^{6} \mathrm{Su}$ trabajo consistió en la atención de la "salud física y moral" de los niños y la provisión de alimentos, ropa, guardapolvos y otros útiles escolares. Para ello, la CNAE promovió la asociación en post de la educación y las cooperadoras escolares (Cosse, 2005, p. 4). Con Perón en el poder, el rol de las cooperadoras en la ayuda escolar no se modificó. El gobierno mantuvo y acrecentó su labor pero encuadró las tareas en el marco de su propia narrativa: las postergaciones de la infancia no debían repararse por caridad o solidaridad sino por principio de justicia social.

La irrupción de las masas implicó un reposicionamiento de las instituciones. Según Arizaga, el CNE debía realizar una labor "no solo en la democratización del futuro ciudadano o en los alcances alfabetizadores sino en la acción periescolar que el gobierno asign [ó] a los establecimientos de educación, como mediadores entre los derechos del niño y los deberes de la familia" (Arizaga, 1948, p. 21). El maestro "debía salir del estrecho recinto del aula y extender su radio de influencia" (Programas de Educación Primaria, 1948, p 22), debía responder a una realidad con "problemas de carácter social [que necesitaban] una solución más acorde con los momentos que se vive” (Exp. 27.747, CNE, 1948). En ese contexto, y con el objetivo de promover "una modificación grande en la forma y en el espíritu de la escuela" (El Monitor de la Educación Común, 1948, N 905-908, p. 87), el CNE vertebró dos líneas de acción destinadas a los niños y a la comunidad: la incorporación de los cursos especiales en quinto y sexto grado y la puesta en marcha de actividades socioculturales destinadas al alumnado y su entorno familiar.

\section{EsPACIOS INSTITUCIONALES DESTINADOS A LOS NIÑOS Y A LA FAMILIA: LAS ACTIVIDADES PERIESCOLARES}

La Secretaría de Didáctica del CNE, a cargo del maestro y médico Luis Giordano y del profesor Prudencio Oscar Tolosa, impulsó medidas para cumplir con el propósito de convertir a la escuela en un centro de irradiación y atracción cultural y "extender su radio de influencia a un círculo tan grande que pueda abarcar toda la actividad del niño” (Arizaga, 1948, p. 21). ${ }^{7}$ Según las autoridades, la obra escolar tenía que trascender los límites del aula porque "la función social que compete a la escuela y que está obligada a realizar...no puede reducirse a la diaria tarea de enseñar. Es mucho más amplia y para cumplirla 
es necesario desplegar una intensa actividad social” (Exp. 22293-C-1948. Archivo Intermedio. AGN). El propósito se materializó en 1948 con la realización de cuatro acciones concretas, las Asociaciones para Padres, los cursos de Pedagogía Familiar, los cursos de pre-aprendizaje y los Clubes Escolares.

\section{Asociaciones para Padres y cursos de Pedagogía Familiar}

Durante los primeros años peronistas, el Estado retomó las acciones de intervención en el ámbito familiar que habían comenzado a instalarse desde los primeros años del siglo XX. En el marco de la proyección que tuvieron los discursos en torno a la maternidad, la importancia que habían adquirido las teorías pedagógicas que ubicaban al niño en el centro de la escena, la profesionalización de la asistencia social, el peso de una nueva sensibilidad en torno a la protección de la infancia y la importancia de la estabilidad del núcleo familiar en el orden social, el Estado aprovechó el organigrama del sistema educativo con el objetivo de canalizar la ayuda social y unificó las acciones destinadas a los niños "comunes" y a la infancia en riesgo o "carenciada" (Cosse, 2005, p. 3-4). A modo de ejemplo, durante los años veinte los controles médicos sobre los escolares, la propaganda e instrucción sanitaria a través del envío de cartillas o volantes informativos, las charlas de las Visitadoras de Higiene, las conferencias radiotelefónicas y proyecciones de películas y diapositivas, habían sido promovidas por los organismos que dependían del CNE con el fin de institucionalizar una verdadera conciencia higiénica en la escuela y el hogar (Cammarota, 2017). Con la llegada de Perón a la presidencia, el sistema de enseñanza primaria siguió siendo una herramienta clave en la colaboración con los servicios asistenciales estatales y ocupó un rol protagónico en las medidas gubernamentales destinadas a la familia.

Desde la escuela, el Estado aspiró a transformar las pautas tradicionales o populares de cuidado infantil. La mayor preocupación, señala Karina Ramacciotti, eran las familias pobres. Dentro de los males sociales, el gobierno interpretaba que el abandono, la mala alimentación y el alcoholismo impulsaban a los niños a la calle, una escuela de delincuencia y mala vida. Frente a ese panorama desolador las autoridades sanitarias pensaron dispositivos para alejar a la infancia del influjo perjudicial de las familias (2010, p. 183). Con un fin similar, pero sin personal técnico especializado, en 1948 el CNE fundó las Asociaciones de Padres y la Campaña de Pedagogía Familiar.

Durante las primeras décadas del siglo XX, el magisterio había expresado su preocupación por la acción social de la escuela. Los registros narran desde la formación de asociaciones escolares avaladas por el CNE con fines caritativos y moralizantes (Bontempo, 2012, p. 214) o el caso de una directora autorizada para constituir una sociedad de protección a los niños pobres (Memoria de la Escuela, Escuela $\mathrm{N}^{\circ} 17$ C.E. $20^{\circ}$ ), hasta la publicación en 1922 en El Monitor de la Educación Común, de un artículo que señalaba "el maestro debe luchar por acercar el hogar a la escuela, para reducir la resistencia del padre, para atraerlo al aula, conversar con él, sacar a la escuela de los límites de las cuatro paredes, para llevar a las familias obras prácticas peri y post escolares" (Picarel, 1922, p. 300).

En 1934 se crearon las Asociaciones para Padres. Ese año el CNE aprobó la experiencia escolar que se desarrolló en la escuela $\mathrm{N}^{\circ} 10$ del C.E $8^{\circ}$ y en 1938 en la No 1 del C.E. $12^{\circ}$ de la actual ciudad de Buenos Aires. El proyecto consistió en encuentros mensuales sistematizados entre los maestros y las familias con el fin de reforzar los vínculos entre la escuela y el hogar. Eran reuniones planificadas por el docente de cada grado para conversar sobre la importancia de la educación moral, intelectual y física de los niños, y para entregar a cada familia un informe individual que detallaba los aspectos integrales de cada alumno (Giordano, 1947, p. 25-30).

La segunda estrategia consistió en el programa Pedagogía Familiar. Su funcionamiento tuvo origen en 1943, cuando el maestro y médico Luis Giordano publicó Pedagogía familiar. Nociones al alcance de todos los padres para la educación integral de los hijos. ${ }^{8}$ En el prólogo de la primera edición, el reconocido intelectual Juan Mantovani había destacado el contenido de la obra y una de las ideas principales, "la propia escuela debe 
llegar con su influencia hasta los hogares, para combatir falsas visiones y absurdas actitudes, que conspiran involuntariamente contra el desarrollo orgánico y psíquico de los hijos" (Giordano, 1946, p. 7).

Según consta en el registro de la escuela $16^{\circ}$ C.E $3^{\circ}$, entre 1947 y 1948, Giordano auspició la creación de reuniones de padres con motivo de las clases de Pedagogía Familiar. Ese último año, el proyecto se llevó a la práctica con el nombre Campaña de Pedagogía Familiar y trató temas supervisados por la Secretaría de Didáctica, sin personal técnico especializado y dictados por las maestras. Se ofrecieron conferencias sobre "La Familia y la educación de los hijos", "La religión y la educación de los hijos", "Factores que condicionan la asimilación de conocimientos", "Niños insuficientes", "La salud como base de la educación infantil", "Valor educativo del ejemplo", "El castigo corporal y sus consecuencias", "La importancia de la educación pre-escolar", entre otros títulos (Reseña Histórica. Escuela $\mathrm{N}^{\circ} 8$ C.E. $6^{\circ}$ ). En otras instituciones, las charlas versaron sobre normas higiénicas, arreglo de la casa, alimentación sana y organización de las compras, obras literarias y preparación cívica para el ejercicio del sufragio femenino. En la escuela $\mathrm{N}^{\circ} 23$ C.E. $16^{\circ}$ se denominó Plan de conferencias de extensión cultural y acción social y abarcó, entre otros temas, "Importancia técnica y Social de las Escuelas de Perfeccionamiento Obrero", "Los derechos del trabajador", "La Antártida Argentina" o "Las Islas Malvinas". En la escuela $N^{\circ} 9$ C.E. $3^{\circ}$ durante diez sábados el personal docente dictó cursos destinados a los padres con el objeto de explicar los métodos educacionales.

\section{Cursos de pre-aprendizaje y Clubes Escolares}

El pre-aprendizaje consistió en la implantación del aprendizaje general en 5 to y 6to grado de la escuela primaria. A diferencia de las Misiones Monotécnicas y de Extensión Cultural de Residencia Transitoria, establecimientos educacionales de capacitación técnica y cultural destinados a adolescentes de zonas rurales del país, ${ }^{9}$ el decreto establecía que

"La incorporación del pre-aprendizaje, no significa introducir en la escuela primaria, la enseñanza de oficios, sino informar a los alumnos sobre prácticas rudimentarias aplicables a todas las actividades, iniciarlos en el manejo de instrumentos, capacitar sus manos para el hacer y lograr que alcancen a poseer una intuición de la técnica, que al egresar de la escuela encontrarán aplicada en la vida diaria" (Decreto 1.100, 1948, art. 2, inc. d).

Según la norma, las actividades debían tener una "íntima conexión con la cultura general”, ser consideradas un recurso educativo y despertar la actividad creadora en el alumno (Decreto 1.100, 1948, art. 2, inc. b). El objetivo consistió en ejercitar el trabajo manual de los niños de acuerdo a su sexo, edad e intereses. La nueva revista oficial que editaba el CNE señaló

"nada de lo que el pre-aprendizaje quiere introducir en nuestra escuela entraña novedad desde el punto de vista pedagógico. Son los principios clásicos, actualizados modernamente por las que a sí mismas se llaman escuelas nuevas. Desde luego que no se toman de esas escuelas las posturas extremas o disolventes, sino aquellos elementos tradicionalmente aceptados por la pedagogía...aunque muy a menudo relegados" (Guión, ductor itineris, 1, 1948, p. 32).

El decreto 1.100 establecía que se debían practicar en horarios complementarios de dos horas como mínimo y en un turno distinto al de la enseñanza graduada. Estaban a cargo de los maestros de los cursos comunes, quienes decidían según sus gustos e intereses el contenido de los talleres. El docente tenía la libertad para ejercer las actividades que resultaran compatibles con las condiciones del aula y los elementos disponibles pues "no se [quería] la uniformidad en las realizaciones, sino la unidad de espíritu en la orientación general del trabajo escolar" (Guión, ductor itineris, 1, 1948, p. 33). El Programa de Instrucción Primaria sugería trabajos de carpintería, metalistería, electrotécnica, cartonado y encuadernación, modelado y construcción, cestería y talabartería, aeromodelismo y juguetería (Figura 1). En el tópico Educación para el hogar, sugerían trabajar con la preparación de alimentos, estudiar sus valores nutritivos, cálculo de cantidades, conservación, economía y vestidos, elementos de corte y confección, amueblamiento y decorado de la casa familiar, higiene 
del hogar y personal, primeros auxilios y puericultura (1949, pp. 62-67). La propuesta se completó con la apertura de actividades a la comunidad.

\section{FIGURA 1}

Construcción de juguetes en la escuela $\mathrm{N}^{\circ} 26$ C.E. $16^{\circ}$. Archivo

Dr. Láinez. Caja 16. Biblioteca Bernasconi. Capital Federal.

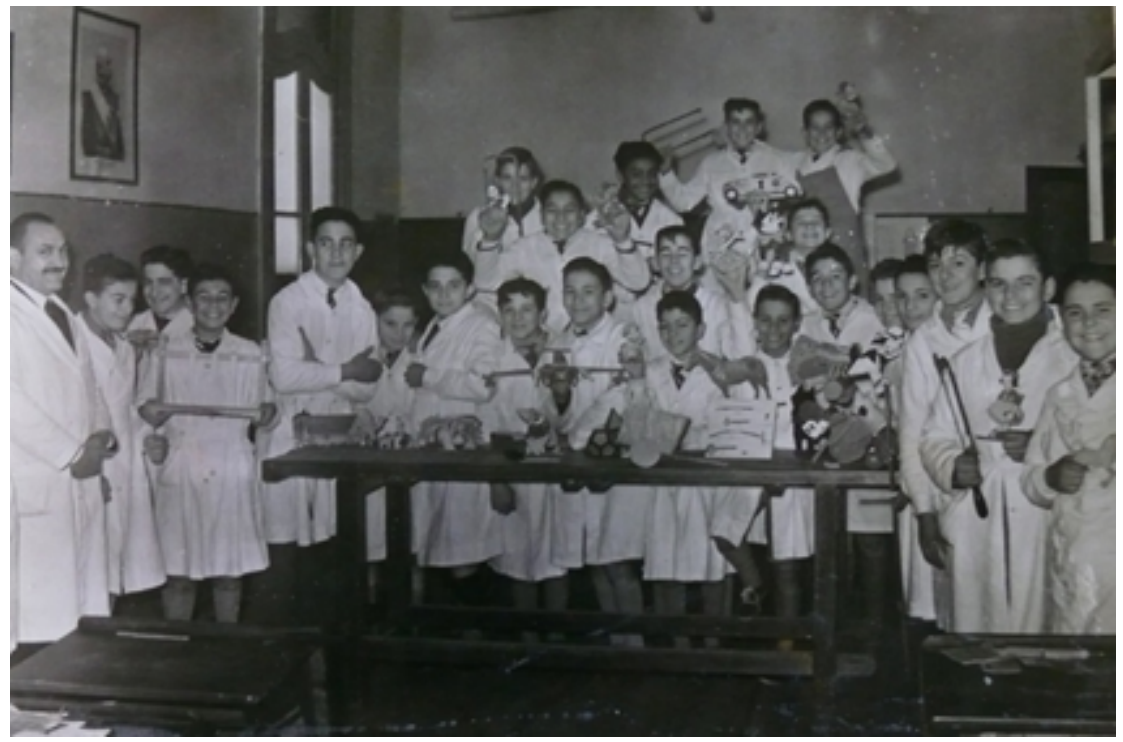

El programa Clubes Escolares consistió en el funcionamiento de actividades culturales, artísticas y deportivas en horarios extra-escolares. ${ }^{10}$ A nivel oficial, el programa de 1936 había propuesto la formación de clubes infantiles de asistencia y ayuda social, de juegos, de lectura y de estudio, de paseos, de reuniones para debatir intereses, de redacción de diarios o revistas, de exposición de trabajos, ejercicios físicos y actividades estéticas, como parte de las formas didácticas para aplicar en las escuelas (Programas para las escuelas comunes de la Capital Federal, 1936, p. 4). Con el cambio curricular de 1939, el proyecto perdió fuerza y desapareció.

Desde diferentes perspectivas, maestros y funcionarios justificaron los obstáculos para su implementación. Para Enrique Stieben, maestro de una escuela pampeana, esos pequeños mundos donde los niños expresaban sus aptitudes, gustos y preferencias "no [habían] sido valorados aún en toda su importancia, pues fueron considerados siempre como simples elementos accesorios...pero nunca como elementos principales" (Stieben, 1940, p. 51). Según los funcionarios del CNE, las actividades propuestas no eran aplicables porque no contaban con personal y demandaban gastos que excedían la posibilidad de la generalidad de los padres (Programa de Instrucción Primaria, 1939, p. 3). Nueve años después, las autoridades educativas retomaron el proyecto y lo adaptaron a la realidad escolar.

En 1948, el gobierno recuperó las experiencias como parte de las acciones del Estado en la ampliación de los derechos del niño. Siguiendo a Isabella Cosse, estos espacios ofrecían a los niños pobres actividades practicadas por los sectores medios

"Así, el presente peronista no sólo se asociaba a la superación de la discriminación en las políticas del Estado sino también a la democratización del acceso a la recreación...En este sentido, en sus discursos el peronismo ofrecía a las madres y los padres humildes la posibilidad de que sus hijos disfrutaran del estatus infantil, evitándoles los problemas y las angustias de los mayores, como merecía cualquier niño, con independencia del origen social de sus progenitores" (2018: 208).

Las actividades se desarrollaron una o dos horas durante la semana, los sábados y, en el caso de la escuela $\mathrm{N}^{\circ} 11$ C.E. $20^{\circ}$, los domingos por la mañana "no pudiendo hacerlo en otros días por funcionar en el local la escuela de Adultos". El ensayo, a cargo de directores y maestros, ofrecía un "amplio campo de actividad cultural y artística" con el propósito de "atraer a miles de niños hacia la escuela, sacándolos del peligro de la 
calle [, y brindar] una oportunidad para que los padres y vecinos se reúnan" (Educación Común en la capital, provincias y territorios nacionales, 1950, p. 14). Desde la perspectiva pedagógica, los alumnos se agruparon según sus intereses o afinidades.

Los Clubes estuvieron conformados por la biblioteca infantil, el museo, niños jardineros, deportes, comedores escolares, teatro escolar, Cruz Roja Infantil, agrupaciones musicales, ropero escolar y Asociaciones de Ex Alumnos. Según la resolución, su propósito era

“a) Hacer de cada establecimiento de enseñanza el centro de la vida cultural y social del barrio o zona donde está ubicado b) Propender a la vinculación afectiva y estrecha de los vecinos con el personal directivo y docente de los establecimientos de enseñanza c) Afirmar y consolidar el espíritu de la Nueva Argentina sobre la base de la unión, de la comprensión, de la solidaridad y de la tolerancia" (Reseña Histórica. Escuela Nº 8 C.E. $6^{\circ}, 1949$ )

Según la reseña de la escuela $\mathrm{N}^{\circ} 16$ C.E. $3^{\circ}$ el club se inauguró "con el objeto de proyectar en el barrio y en el hogar, la acción educativa, benéfica y social de la escuela". Otras, anunciaron que su función consistió en vincular la familia con la institución escolar. El Club, señalaron en la escuela $\mathrm{N}^{\circ} 11$ C.E. $17^{\circ}$, "sustrae de la calle a muchos niños, proporcionándoles lugar seguro. En él está protegido, dirigido y con diversos entretenimientos, unos con fines útiles y otros puramente de esparcimiento para horas felices". La escuela No12 C.E. $9^{\circ}$ aclara en el ítem "Acción Social y Cultural”, que durante más de treinta y tres años de vida, "siempre" unió a su función educativa la acción social y cultural, que "en todo momento, se preocupó por la educación integral de los niños, participó de sus inquietudes y trató de conocer los problemas del hogar, extendiendo su acción a ellos".

La escuela $\mathrm{N}^{\circ} 2$ C.E. $3^{\circ}$ incorporó en su reseña una perspectiva pedagógica ausente en la mayor parte de los documentos analizados

"los alumnos adhieren entusiasmados a la tarea, que para el docente recibe la denominación de peri-escolar, pero que en la mente del educando adquiere la trascendencia de los actos libres, ya que la influencia que sobre ellos ejerce el personal docente es de simple indicación, permitiendo que los niños eduzcan [sic] sus propias capacidades y estimulando condiciones que se descubren durante las horas de esparcimiento" (Escuela Fray Cayetano. Su Historia, 1949).

Las apreciaciones sobre el éxito de la propuesta no fueron uniformes. ${ }^{11}$ En la escuela de adultos $\mathrm{N}^{\circ} 7$ C.E. $6^{\circ}$, el narrador comenta que, según las opiniones de las maestras que figuran en un acta de 1950, este club escolar no tuvo éxito

"los niños que concurren son en su mayoría de escuela diurna, hay entusiasmo en los juegos al aire libre y en los programas
cinematográficos. Solo algunos alumnos de los grados inferiores y algunos pequeños del barrio, no el alumnado adulto por
razones de horario y ocupaciones diversas, concurren al club. En resumidas cuentas, el alumnado adulto no puede concurrir
a ese horario por lo que el funcionamiento del club escolar no cumple los fines perseguidos al implantarlo" (Historia de la
escuela, 1950).

La reseña de la escuela $\mathrm{N}^{\circ} 4$ C.E. $17^{\circ}$ señala que la asistencia fue variable, según la directora, porque no era obligatorio. En la escuela No23 C.E. $19^{\circ}$ el relator indicó

“funcionó entusiasta los primeros tiempos del verano pero luego decayó el interés y fue interrumpido por falta de asistentes en las épocas inclementes y de frío...mientras la escuela no cuente con el toldo en los días destemplados...no podrá funcionar el club y de esta manera precaria, el interés general de los niños del barrio se pierde" (Reseña histórica, 1949).

Con respecto a las instalaciones, la escuela No17 C.E. $19^{\circ}$ menciona que el Club Escolar tuvo lugar en un edificio "que sólo cuenta con los bancos de clase como asientos" y la escuela №16 del mismo distrito expresa que debió reducir una hora en invierno "debido al lugar muy frío y a la distancia de la escuela a los hogares”. Los problemas edilicios fueron el principal obstáculo que debieron enfrentar y frustraron el objetivo oficial de extenderlos durante el receso de verano. Renombrados Vacaciones Útiles, se implementaron con el propósito de contener a los escolares que "viven en la calle, en muchos casos en una situación de franco abandono"(Educación Común en la capital, provincias y territorios nacionales, 1950, p. 17). En la escuela $\mathrm{N}^{\circ}$ 
9 del C.E. $17^{\circ}$, en Vacaciones Útiles se festejó la fiesta de la Concepción de la Virgen, se celebró la primera comunión de los niños, y para Navidad, se repartieron golosinas y juguetes. ${ }^{12}$

Los Clubes estaban dirigidos por el personal directivo y cada institución eligió a los responsables de los talleres dictados. Muy pocos documentos señalan la presencia de profesores especializados en actividades periescolares, la mayor parte de las escuelas contaron con la buena voluntad y predisposición de todo el personal, padres, alumnos y el vecindario en general.

Los informes contienen escasa información sobre el contenido específico de los talleres, los criterios de selección que aplicaron los alumnos y alumnas para elegirlos o la diferenciación por género. En conjunto, las fotografías que acompañan la descripción sobre los clubes retratan escenas donde los protagonistas son los niños y las niñas, en espacios abiertos, con movimientos espontáneos, en pose, desordenados y risueños, en contacto directo con la naturaleza en patios amplios o espacios pequeños (Figura 2).

FIGURA 2

Niños del Club de Jardineros de la escuela $N^{\circ} 26$ C.E. $16^{\circ}$. Archivo Dr. Láinez. Caja 16. Biblioteca Bernasconi. Capital Federal

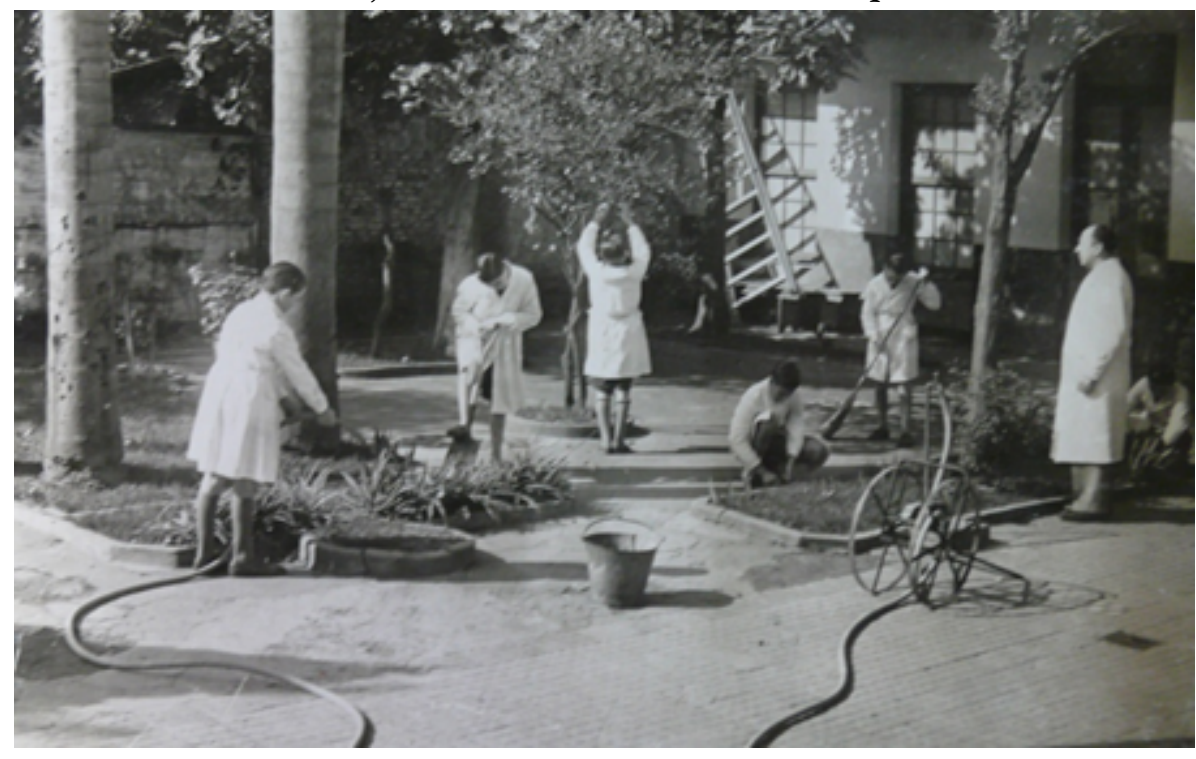

Los relatos e imágenes demuestran que participaron de clubes de lectura de cuentos, teatro de títeres, charlas literarias, recitación de conjunto, poesías, comedias, teatro infantil, dibujo, uso del tocadiscos, rondas escolares, adivinanzas, audiciones artísticas, banda rítmica y coro, visitas al circo o a funciones de teatro infantil, cursos sobre seguridad en el tránsito, museo, periodismo, transmisiones radiales y juegos deportivos.

Con respecto a las actividades físicas, las fotografías muestran ejercicios simples combinados con juegos de patio, deportes colectivos, rondas escolares, excursiones y actividades al aire libre (Figura 3). 
FIGURA 3

Club Escolar de la escuela $\mathrm{N}^{\circ} 12$ C.E. $16^{\circ}$. Excursión a Olivos

Archivo Dr. Láinez. Caja 16. Biblioteca Bernasconi. Capital Federal

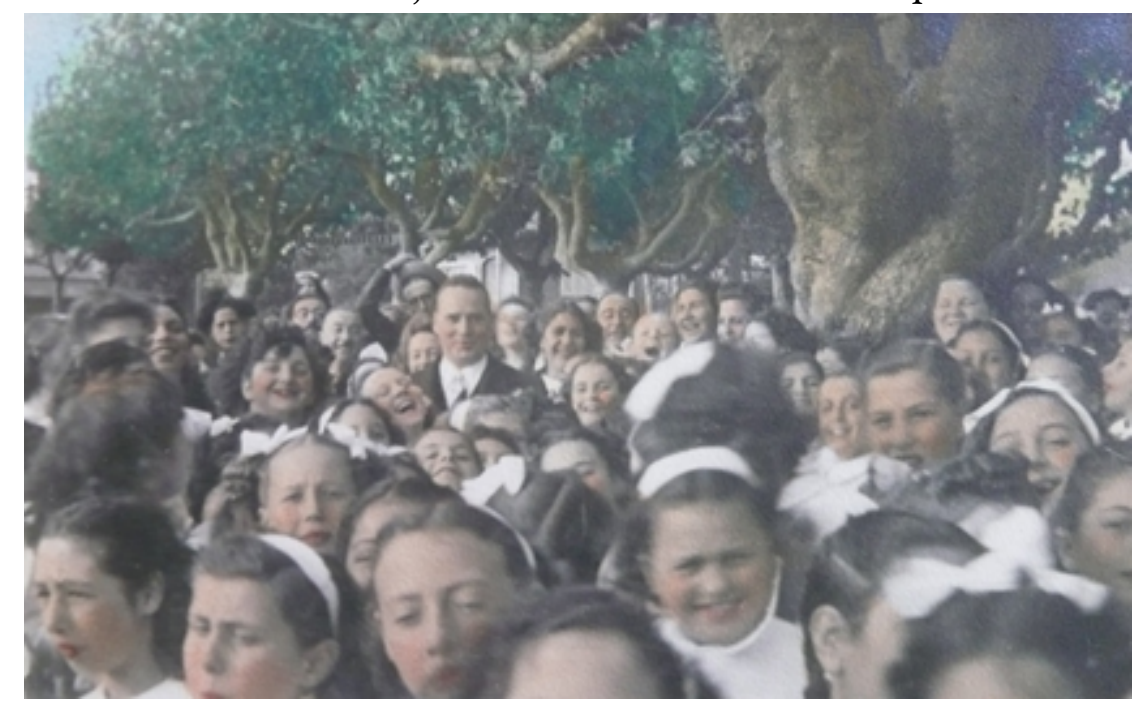

En algunos casos, como en la escuela $\mathrm{N}^{\circ} 26$ C.E. $16^{\circ}$, los alumnos realizaban en el taller de pre-aprendizaje elementos que luego utilizarían en el Club (Figura 3). En esa escuela también funcionó el Club Cultural Católico y el Club Folklórico y Tradicionalista, Club de Madres y de Aeromodelismo.

La biblioteca conformó una de las comisiones más exitosas de los Clubes, la mayor parte de ellos recibía la colaboración de la Asociación de ex alumnos y de la Cooperadora para la provisión de libros, obras clásicas adaptadas a la literatura infantil, vidas ejemplares, viajes y costumbres y cuentos infantiles. Para las funciones cinematográficas, las películas eran distribuidas por el Ministerio de Marina, la Caja Nacional de Ahorro y el Cine Escuela Argentino. La escuela $\mathrm{N}^{\circ} 17$ del C.E. 16 señala que hicieron conocer el suelo patrio con sus bellezas, riquezas e industrias "pasándose películas documentadas en donde se puede admirar la gran obra de reconstrucción y recuperación de nuestra nueva Argentina”.

La labor realizada por las cooperadoras escolares fue fundamental para la concreción de las iniciativas decretadas por la reforma. Estas asociaciones continuaron trabajando en la dotación de calzado, ropa, útiles, meriendas y bibliotecas. En 1950, el gobierno reconoció su invalorable actuación en la mantención de la "miga de pan", la "copa de leche" y el aporte de materiales para los Clubes Escolares y Vacaciones Útiles (Educación Común en la capital, provincias y territorios nacionales, 1950, p. 16). El Club de la escuela No6 C.E. $17^{\circ}$, por ejemplo, funcionó gracias a las donaciones de vecinos y de un auxiliar que construyó un biombo para el teatro de títeres. En la escuela $\mathrm{N}^{\circ} 20$ C.E. $5^{\circ}$, la Asociación de ex alumnos ofreció una función teatral para juntar fondos, en la escuela $\mathrm{N}^{\circ} 12$ C.E. $17^{\circ}$ la Asociación Cooperadora acondicionó un terreno, juegos de plaza, arenero, juegos educativos, mesa de ping-pong, mesas y bancos, y donó escenarios, discos para la enseñanza de folklore, equipos de radio, altoparlantes y micrófonos, equipos transmisores de radiotelefonía, toca discos y escenarios. Todas las escuelas agradecen en sus informes el trabajo realizado por la cooperadora, la Asociación de ex alumnos, las madres y el personal docente en la recolección de los materiales, los aparatos y los muebles para los juegos deportivos y artísticos.

Las actividades periescolares desarrollas durante los primeros años peronistas componen uno de los niveles de acción del Estado en el cual se vinculó la reforma pedagógico-social con el proyecto de democratización de la cultura y protección de la niñez. Siguiendo a Donna Guy, entendemos que estas acciones fueron construidas alrededor de políticas sociales más tempranas que ofrecieron de manera desarticulada aunque efectiva, el edificio compuesto por subsidios nacionales y el trabajo de los grupos filantrópicos desde comienzos del siglo XX (2011, p. 27). Desde una mirada romántica del trabajo docente, el CNE recurrió 
al altruismo de las asociaciones cooperadoras y a la vocación de servicio del magisterio, para cubrir el déficit edilicio y la ausencia de personal técnico especializado y de materiales didácticos. La experiencia reconstruida abre el camino para profundizar el rol de las mujeres en estas actividades y repensar los límites que enfrentaron las políticas socio-escolares del gobierno peronista en su período de gestación.

\section{A MODO DE CIERRE}

La recuperación de los argumentos y prácticas de intervención del Estado en la comunidad permite articular las experiencias de acción periescolar impulsadas por el peronismo con los mecanismos de acción socio-pedagógica ensayados en períodos anteriores. Durante el breve período analizado, el gobierno apeló a la dinámica previa de ayuda social y la insertó en un entramado discursivo que justificó el compromiso y obligación del Estado en la protección de la infancia y la familia a través del concepto de justicia social.

Las estrategias destinadas a asistir al niño-alumno y su familia se construyeron dentro de la estructura del sistema. Si bien fueron incluidas en los decretos de la reforma educativa para la enseñanza primaria, la urgencia en la aplicación y la red de instituciones administrativas aún en formación, obstaculizaron el éxito del proyecto. La ausencia de infraestructura específica, la dependencia de la labor de las cooperadoras en el financiamiento del material didáctico y la ausencia de personal técnico, demostraron los alcances y límites de una actividad fugaz que finalizó en 1950 cuando los recortes presupuestarios atentaron contra su funcionamiento y determinaron su cierre.

\section{REFERENCIAS}

Armus, D. (2007). La ciudad impura. Salud, tuberculosis y cultura en Buenos Aires, 1870-1950. Buenos Aires: Edhasa.

Aversa, M. M. (2008). La política asistencial hacia la infancia popular: modelando el futuro peronista (1946-1955). Primer Congreso de Estudios sobre el Peronismo, Mar del Plata. Recuperado de http://redesperonismo.com.ar/ biblioteca/actas-del-1er-congreso/ MardelPlata.

Bernetti, J. L., y Puiggrós, A. (2006). Peronismo: cultura politica y educación (1945-1955). Buenos Aires: Galerna.

Biernat, C., y Ramacciotti, K. (2013). Crecer y multiplicarse. La politica sanitaria materno-infantil. Argentina 1900-1960. Buenos Aires: Biblos.

Billorou, M. J. (2017). Niños sanos para el progreso pampeano. Las políticas de protección de la salud infantil en el Territorio Nacional de La Pampa en la primera mitad del siglo XX. Historia Caribe. 12 (31), 45-84.

Bontempo, P. (2012). Los niños de Billiken. Las infancias en Buenos Aires en las primeras décadas de siglo XX. Anuario del Centro de Estudios Históricos "Prof. Carlos S. A. Segreti". (12), 205-221. Recuperado de https://cehsegreti.o rg.ar/archivos/FILE_00000408_1457723346.pdf

Bordagaray, M., y Gorza, A. (2010). Socialización política y de género de la infancia durante el primer peronismo a través de la revista mundo infantil (1950-1952). En C. Panella, y G. Korn (Comp.), Ideasy debates para la nueva Argentina: revistas culturales y politicas del peronismo: 1946-1955 (pp. 255-281). La Plata: Universidad Nacional de La Plata.

Cammarota, A. (2010). El cuidado de la salud de los escolares en la provincia de Buenos Aires durante el primer peronismo (1946-1955). Las libretas sanitarias, las fichas de salud y las cédulas escolares. Propuesta Educativa, (35), 113-120. Recuperado de http://www.propuestaeducativa.flacso.org.ar/archivos/jovenes/12.pdf

Cammarota, A. (2017). Saberes médicos y medicalización en el ámbito escolar (1920-1940). Revista Pilquen. Sección Ciencias Sociales.19 (3), 33-51. Recuperado de http://revele.uncoma.edu.ar/htdoc/revele/index.php/Sociales/ article/view/1417

Carli, S. (2002). Niñez, pedagogía y política. Transformaciones de los discursos acerca de la infancia en la historia de la educación argentina entre 1880 y 1955. Buenos Aires: UBA: Miño y Dávila.

Carli, S. (2005). Los únicos privilegiados son los niños. Todo es Historia, (457), 58-65. 
Cosse, I. (2005). La infancia en los años treinta. Todo es Historia, (457), 48-57.

Cosse, I. (2006). Estigma de nacimiento: Peronismo y orden familiar 1946-1955. Buenos Aires: Fondo de Cultura Económica/UDESA.

Cosse, I. (2018). La primacía de la infancia y la naturaleza de los vínculos filiales. En L. Lionetti, I. Cosse, y M. C. Zapiola (Comp.), La historia de las infancias en América Latina (pp. 199-224). Tandil: Universidad Nacional del Centro de la Provincia de Buenos Aires.

Cosse, I., Llobet, V., Villalta, C, y Zapiola M.C. (Ed.) (2011). Infancias: politicas y saberes en Argentina y Brasil : siglos $X I X y X X$. Buenos Aires: Teseo.

Ferreyra, G. (2019). Educación primaria y peronismo: trayectoria de los agentes estatales que intervinieron en la primera reforma educativa (1948-1949). Historia de la Educación. Anuario. 20(1), 162-179. Recuperado de htt p://ppct.caicyt.gov.ar/index.php/anuario/article/view/15222

Girbal-Blacha, N. (2006). La Argentina peronista y 'los únicos privilegiados. En H. Biagini y A. Roig (Dir.), El pensamiento alternativo en la Argentina del siglo XX. Obrerismo, vanguardia, justicia social (1930-1960) (pp. 305-319). Buenos Aires: Biblos.

Girbal-Blacha, N. (2014). En la argentina peronista "los únicos privilegiados son los niños" (1946-1955). La doctrina desde la biblioteca infantil "General Perón”. Historia Contemporánea, (50), 133-162. Disponible en https://ri.conicet.gov.ar/bitstream/handle/11336/51803/CONICET_Digital_Nro.c6c6f38a-0c7b-49e9-9 17c-0509c620e103_A.pdf? sequence $=28$ isAllowed $=\mathrm{y}$

Guy, D. (2011). Las mujeres y la construcción del Estado de Bienestar. Caridad y creación de derechos en Argentina. Buenos Aires: Prometeo.

Leonardi, Y. (2010). Educación y entretenimiento para los niños peronistas: la infancia como cuerpo político (1946-1955). VI Jornadas de Sociología de la UNLP. Universidad Nacional de La Plata. Facultad de Humanidades y Ciencias de la Educación, La Plata. Recuperado de http://www.memoria.fahce.unlp.edu.ar/tra b_eventos/ev.5115/ev.5115.pdf

Lionetti, L. (2009). La cuestión social en torno a los niños pobres. Las estrategias conjuntas de la escuela pública y las instituciones particulares en la primera mitad del siglo XX. En IV Jornadas de Historia Politica. Universidad Nacional del Sur. Recuperado de www.historiapolitica.com/datos/biblioteca/4jornadas/lionetti.pdf

Lionetti, L. y Míguez, D. (2010). Las infancias en la historia argentina. Intersecciones entre prácticas, discursos e instituciones (1890-1960). Rosario: Prohistoria ediciones.

Lionetti, L., Cosse, I. y Zapiola, M. C. (Comp.) (2018). La historia de las infancias en América Latina. Tandil : Universidad Nacional del Centro de la Provincia de Buenos Aires.

Llobet, V. (comp.) (2013). Pensar la infancia desde América Latina : un estado de la cuestión. Buenos Aires: CLACSO.

Lvovich, D., y Suriano, J. (2006). Las políticas sociales en perspectiva histórica. Argentina, 1870-1952. Buenos Aires: Prometeo.

Moreno, J. L., y Cacopardo, M. C. (2005-2006). Familia, pobreza y desamparo antes y durante el primer gobierno peronista, 1935-1955. Población y Sociedad, (12-13), 91-131. Recuperado de https://dialnet.unirioja.es/servlet /articulo?codigo $=3265440$

Petitti, E. M. (2017). Más allá de una escuela peronista. Políticas públicas y educación en la provincia de Buenos Aires. Rosario: Prohistoria.

Plotkin, M. (2007). Mañana es San Perón: propaganda, rituales politicos y educación en el régimen peronista (1946-1955). Caseros: Eduntref.

Ramacciotti, K. (2010). De chico, el árbol se puede enderezar. La salud infantil durante el peronismo. En L. Lionetti y Míguez, D. (Comp.), Las infancias en la historia argentina. Intersecciones entre prácticas, discursos e instituciones (1890-1960) (pp. 175-198). Rosario: Prohistoria.

Spregelburd, R. (1997). La enseñanza técnica en el nivel primario. Las Misiones monotécnicas. Análisis de un caso en Luján. En Cucuzza, H. R. Estudios de Historia de la Educación durante el primer Peronismo 1943-1955. Lujan: Editorial Los Libros del Riel. 
Villalta, C. (2003). Entre la ilegitimidad y el abandono: la primera ley de adopción de niños. Cuadernos del Sur, 32, 27-48. Disponible en: http://bibliotecadigital.uns.edu.ar/scielo.php?script=sci_arttext\&pid=S1668-76042003 001100002\&lng=es\&nrm=iso >. ISSN 1668-7604

\section{Fuentes}

“Actuación del Señor Delegado Interventor en el Consejo Nacional de Educación”, El Monitor de la Educación Común, $1948,905-908,87$.

Argentina. Consejo Nacional de Educación. (1936). Programas para las escuelas comunes de la Capital Federal. Buenos Aires: Talleres Gráficos del Consejo Nacional de Educación.

Argentina. Ministerio de Educación. Dirección General de Enseñanza Primaria (1950). Educación Común en la capital, provincias y territorios nacionales 1948. Buenos Aires: Dirección General de Enseñanza Primaria.

Argentina. Ministerio de Justicia e Instrucción Pública. Consejo Nacional de Educación. (1939). Programa de Instrucción Primaria. Buenos Aires: Talleres Gráficos del Consejo Nacional de Educación.

Argentina. Secretaría de Educación. Consejo Nacional de Educación (1949). Programa de Instrucción Primaria. Buenos Aires: Talleres Gráficos del Consejo Nacional de Educación.

Arizaga, J. P. (1947). Aplicación del Plan de Gobierno en la enseñanza primaria. Conferencia pronunciada en el teatro nacional Cervantes el 17 de diciembre de 1947. El Monitor de la Educación Común, 897-900, 35-53.

Arizaga, J. P.(1948). Discurso pronunciado por el Señor Subsecretario de Educación al poner en posesión de sus funciones al Delegado Interventor del Consejo Nacional de Educación. Guión, ductor itineris, 1, 17-16.

Biblioteca Bernasconi. Archivo Dr. Láinez. Caja Nº-18 Capital Federal. C.E. 2º-20․ El Monitor de la Educación Común, 1948, 905-908.

Exp. 22293-C-1948. Archivo Intermedio. AGN.

Giordano, L. (1947). Acción Social de la escuela. Nuevas Asociaciones de padres. Buenos Aires: Kapelusz.

Giordano, L. (1946). Pedagogía familiar. Nociones al alcance de todos los padres para la educación integral de los hijos. Kapelusz y Cía: Buenos Aires.

Picarel, J. (1922). Acción Social del maestro. Recuperado de http://repositorio.educacion.gov.ar:8080/dspace/hand le/123456789/107449

Revista de Educación. Secretaria General Técnica del Ministerio de Educación y Ciencia (1973), №227-228, 185-186.

Stieben, E. (1940). La función de los Clubes Escolares, 49-51. Recuperado de http://repositorio.educacion.gov.ar/ds pace/bitstream/handle/123456789/98588/Monitor_11114.pdf?sequence=1

\section{Notas}

1 El término periescolares hace referencia a las actividades educativas culturales denominadas también pos-escolares o extraescolares, a menudo se dirigen a una población escolarizada y tienen un carácter marginal en relación a la escuela formal. Citado en Revista de Educación. Secretaría General Técnica del Ministerio de Educación y Ciencia (1973).

2 Los documentos analizados pertenecen a las escuelas del C.E. $3^{\circ}$, situadas en Balvanera, Monserrat y Constitución, C.E $5^{\circ}$, en Barracas, C.E. $6^{\circ}$, en San Cristóbal, Boedo y Parque Patricios, C.E. $16^{\circ}$, en Villa Urquiza, C.E. $17^{\circ}$, en Villa Santa Rita, Villa del Parque y Villa Devoto, C.E. $19^{\circ}$, en Vila Lugano y C.E. $20^{\circ}$, en Mataderos.

3 Para los enfoques teóricos y metodológicos de la historiografía argentina y latimoamericana véase, entre otros, Lionetti y Míguez (2010); Cosse, Llobet, Villalta y Zapiola (2011); Llobet (2013); Lionetti, Cosse, y Zapiola (2018).

4 Sobre el adoctrinamiento en las escuelas véase, entre otros, Plotkin (2004).

5 El profesor Jorge Pedro Arizaga fue subsecretario de Instrucción Pública desde 1946 hasta 1948 y subsecretario de Educación desde 1948 hasta 1949. Para su trayectoria véase, entre otros, Ferreyra (2019).

6 El CNE fue el organismo que administró el funcionamiento de las escuelas primarias de la Capital Federal y las escuelas primarias nacionales de las Provincias y los Territorios. En 1943 fue intervenido y desapareció en 1949, cuando fue creado el Ministerio de Educación y la enseñanza primaria pasó a manos de la Dirección General de Enseñanza Primaria. 
7 Luis Giordano y Prudencio Oscar Tolosa fueron secretarios de Didáctica del delegado interventor del CNE Federico Daus durante el breve lapso 1948-1949. El primero era médico y maestro, colaborador de la revista El Monitor de la Educación Común durante la década del treinta y fundador del Consejo Asesor de Pedagogía Familiar. El profesor Tolosa se había desempeñado como maestro, director e inspector. Durante la década del treinta había integrado la Sección Argentina de la Liga Internacional por la Nueva Educación y había co-dirigido la revista La Obra hasta 1947. Sobre sus trayectorias véase Ferreyra (2019).

8 Desde comienzos del siglo XX circulaban libros con recomendaciones sobre la crianza, El libro de las madres de Gregorio Aráoz Alfaro, La Educación del Hijo de Constancio Vigil, o las revistas Hijo Mio! y Madre y Niño, entre otros. Sobre el tema véase Bontempo (2012).

9 Las Misiones monotécnicas fueron creadas por el decreto 20.628, en 1948 pasaron a depender de la Dirección General de Enseñanza Técnica. Para el funcionamiento de las misiones y su relación con la educación técnica oficial véase Spregelburd (1997).

10 Los Clubes Escolares habían sido parte de las propuestas de renovación pedagógica que surgieron en Europa y se instalaron en la Argentina durante la primera parte del siglo XX, funcionaron entre 1924 y 1940 en varias escuelas primarias de la Capital Federal (Bernetti y Puiggrós, 2006, p. 224).

11 Los informes escolares, los registros del CNE y las publicaciones de la Secretaría de Educación de 1948, 1949 y 1950 no incluyen datos sobre la cantidad de inscriptos.

12 En el Boletín de Comunicaciones $\mathrm{N}^{\circ} 101$ de diciembre de 1949 y $\mathrm{N}^{\circ} 102$ de enero de 1950 se nombran designaciones de maestros periescolares para Vacaciones Útiles, sin embargo, en las Memorias o Reseñas Históricas de las escuelas no hallamos referencias sobre estas actividades. 